\title{
Narrowing Band Gap of BiOCl for the Hydroxyl Radicals Generation of Photocatalysis under Visible Light
}

Jianhua Hou ${ }^{*}, \dagger, \ddagger$ Dian Dai ${ }^{\dagger}$, Rui Wei ${ }^{\dagger}$, Xiaoge $W^{\dagger}$, Xiaozhi Wang ${ }^{*}, \dagger, \ddagger$, Muhammad Tahir,$\S$ and Ji-Jun Zou ${ }^{*}, \S$

$\uparrow$ School of Environmental Science and Engineering, Yangzhou University, Yangzhou, 225000, P.R China

$\ddagger$ Jiangsu Collaborative Innovation Center for Solid Organic Waste Resource Utilization, Nanjing, 210095, P.R. China

$\S$ Key Laboratory for Green Chemical Technology of the Ministry of Education,School of Chemical Engineering and Technology, Tianjin University,Tianjin, 300072, P.R.China *E-mail address: jhhou@yzu.edu.cn (J. Hou); xzwang@yzu.edu.cn (X. Wang); jj_zou@tju.edu.cn (J. Zou).

1. Fig. S1. Figure S1. (a) XPS spectra of $\mathrm{Cl} 2 \mathrm{p}$ region and (b) XPS spectra of O 1s region of BiOCl-X samples.

2. Table S1. XPS result of the BiOCl-X samples.

3. Table S2. BET surface area and pore volume of the BiOCl-X samples.

4. Fig. S2. Raman spectra of BiOCl-X samples.

5. Fig. S3. Photographs of BiOCl-X samples before and after the modification of thiourea (TU) $\left(\mathrm{CH}_{4} \mathrm{~N}_{2} \mathrm{~S}=5 \sim 30 \mathrm{~mL}\right)$.

6. Fig. S4. The adsorption rate of $\mathrm{RhB}$ for BiOCl-X samples until reaching to the absorption-desorption equilibrium.

7. Table S3. Comparison of the obtained specific surface area and photocatalysis performance results with literature data of the catalysts.

8. References. 

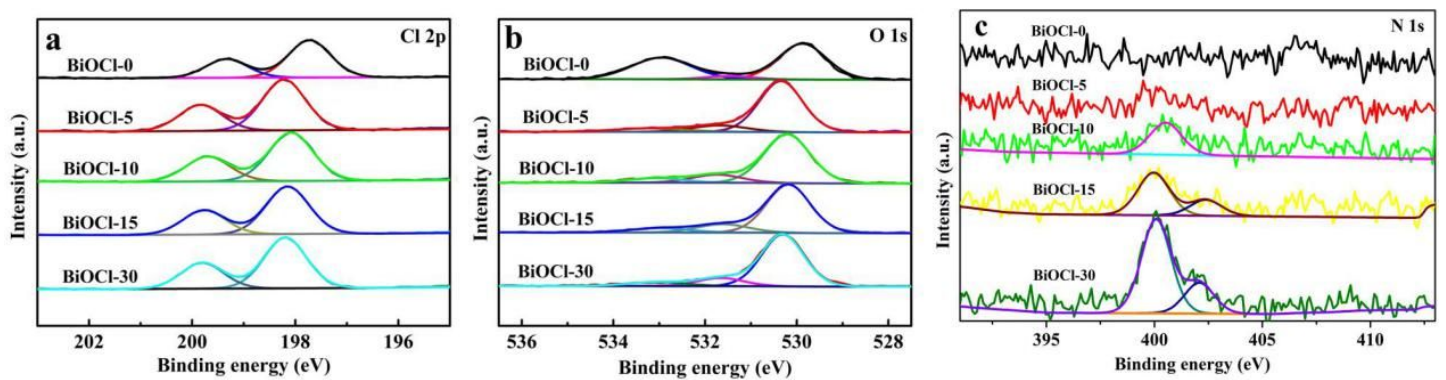

Fig. S1. (a) XPS spectra of $\mathrm{Cl} 2$ p region, (b) XPS spectra of O 1s region and (c) XPS spectra of $\mathrm{N}$ 1s region of $\mathrm{BiOCl}-\mathrm{X}$ samples.

Table S1. XPS result of the BiOCl-X samples.

\begin{tabular}{lccccc}
\hline & Bi 4f & Cl 2p & O 1s & C 1s & N1s \\
\hline BiOCl-0 & 16.16 & 18.19 & 41.12 & 24.53 & - \\
BiOCl-5 & 19.68 & 20.38 & 32.12 & 27.38 & 0.44 \\
BiOCl-10 & 19.48 & 21.12 & 32.96 & 25.15 & 1.29 \\
BiOCl-15 & 21.33 & 20.89 & 33.25 & 22.37 & 2.16 \\
BiOCl-30 & 16.3 & 17.83 & 30.21 & 27.97 & 7.69 \\
\hline
\end{tabular}

Table S2. BET surface area and pore volume of the BiOCl-X samples.

\begin{tabular}{cccccc}
\hline Sample & BiOCl-0 & BiOCl-5 & BiOCl-10 & BiOCl-15 & BiOCl-30 \\
\hline $\mathbf{S}_{\mathbf{B E T}} / \mathbf{m}^{\mathbf{2}} \mathbf{g}^{-1}$ & 4.89 & 39.43 & 56.07 & 42.94 & 43.90 \\
$\mathbf{V} / \mathbf{~ c m}^{\mathbf{3}} \mathbf{g}^{-1}$ & 0.07 & 0.18 & 0.19 & 0.15 & 0.21 \\
\hline
\end{tabular}




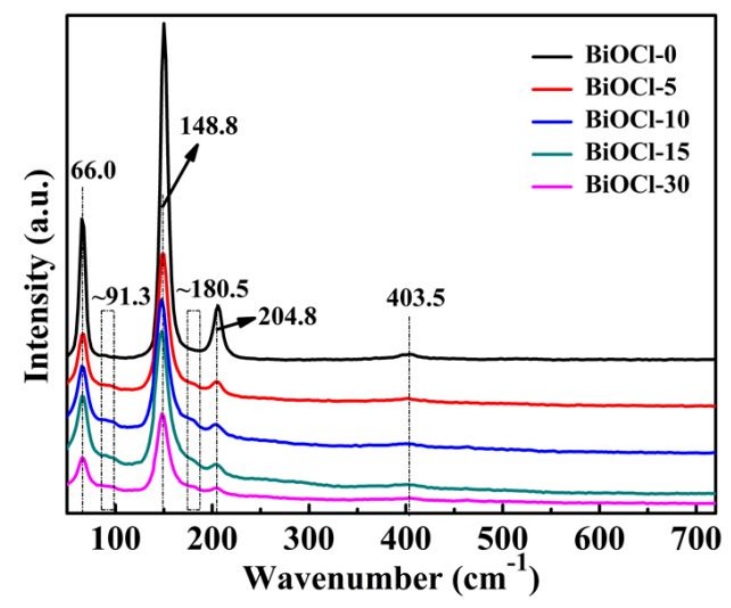

Fig. S2. Raman spectra of BiOCl-X samples.
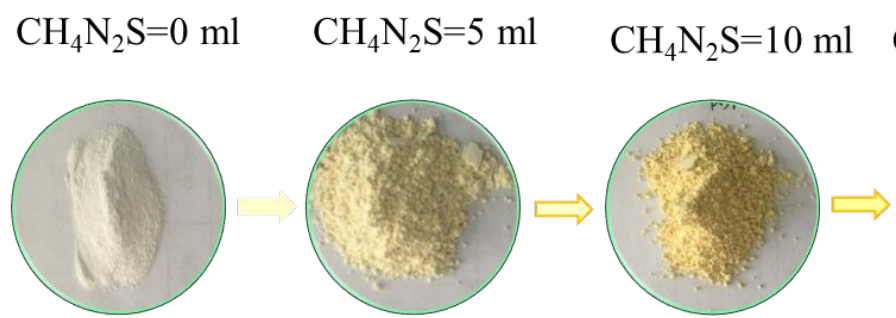

$\mathrm{CH}_{4} \mathrm{~N}_{2} \mathrm{~S}=15 \mathrm{ml}$

$\mathrm{CH}_{4} \mathrm{~N}_{2} \mathrm{~S}=30 \mathrm{ml}$

BiOCl-0

BiOCl-5

BiOCl-10

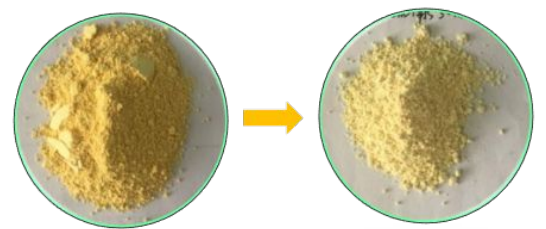

BiOCl-15

BiOCl-30

Fig. S3. Photographs of BiOCl-X samples before and after the modification of thiourea (TU) $\left(\mathrm{CH}_{4} \mathrm{~N}_{2} \mathrm{~S}=5 \sim 30 \mathrm{~mL}\right)$.

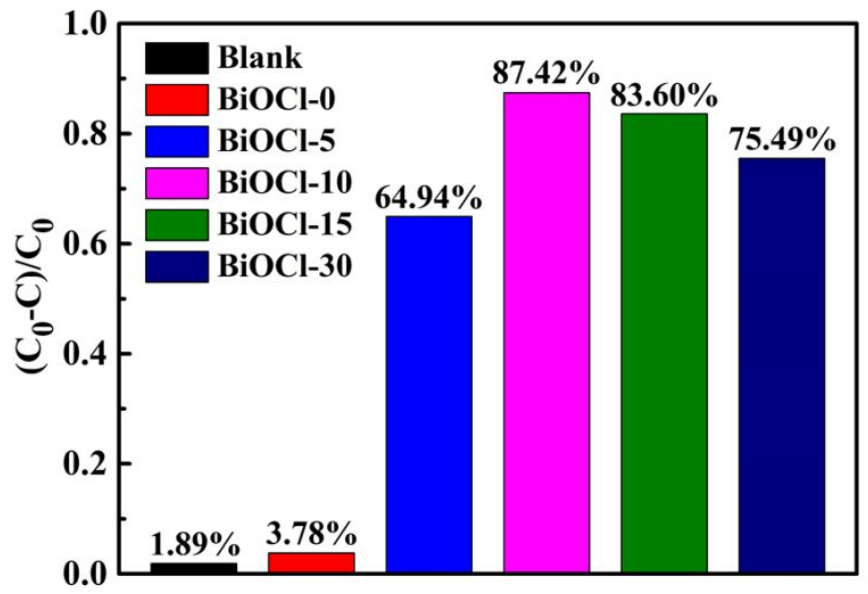

Fig. S4. The adsorption rate of $\mathrm{RhB}$ for BiOCl-X samples until reaching to the absorption-desorption equilibrium. 
Table S3. Comparison of the obtained specific surface area and photocatalysis performance results with literature data of the catalysts.

\begin{tabular}{|c|c|c|c|c|}
\hline Materials & $\begin{array}{c}\mathrm{S}_{\mathrm{BET}} \\
\left(\mathrm{m}^{2} \mathrm{~g}^{-1}\right)\end{array}$ & Simulated pollutant & Degradation rate & Ref. \\
\hline BiOCl-10 & 56.07 & $\begin{array}{c}20 \mathrm{mg} \mathrm{L}^{-1} \text { Tetracycline } \\
20 \mathrm{mg} \mathrm{L}^{-1} \mathrm{RhB}\end{array}$ & $95 \%$ in $8 \mathrm{~min}$ & $\begin{array}{l}\text { This } \\
\text { work }\end{array}$ \\
\hline $\mathrm{t}-\mathrm{Se} / \mathrm{BiOCl}$ & 42.09 & $\begin{array}{c}10 \mathrm{mg} \mathrm{L}^{-1} \text { Tetracycline } \\
\text { hydrochloride }\end{array}$ & $<90 \%$ in $90 \mathrm{~min}$ & 1 \\
\hline $\mathrm{BiOCl} / \mathrm{Bi}_{2} \mathrm{Ti}_{2} \mathrm{O}_{7}$ & 21 & $\begin{array}{c}50 \mathrm{mg} \mathrm{L}^{-1} \text { Tetracycline } \\
\text { hydrochloride }\end{array}$ & $90 \%$ in $120 \mathrm{~min}$ & 2 \\
\hline $\mathrm{BiOBr}$ & 32.0 & 20 mg L-1 Tetracycline & $94 \%$ in $90 \mathrm{~min}$ & 3 \\
\hline $\mathrm{In}_{2} \mathrm{~S}_{3} @ \mathrm{MIL}-125(\mathrm{Ti})$ & 1548.3 & $46 \mathrm{mg} \mathrm{L}^{-1}$ Tetracycline & $63.3 \%$ in $60 \mathrm{~min}$ & 3 \\
\hline p-doped g- $\mathrm{C}_{3} \mathrm{~N}_{4}$ & 22.53 & $10 \mathrm{mg} \mathrm{L}^{-1}$ Tetracycline & 89.7 in $60 \mathrm{~min}$ & 4 \\
\hline $\mathrm{BiOCl}-\mathrm{g}-\mathrm{C}_{3} \mathrm{~N}_{4}$ & - & $10 \mathrm{mg} \mathrm{L}^{-1}$ 4-chlorophenol & $95 \%$ in $2 \mathrm{~h}$ & 5 \\
\hline $\mathrm{BiOI} / \mathrm{BiOCl}$ & 4.2 & $20 \mathrm{mg} \mathrm{L}^{-1} \mathrm{MO}$ & $95 \%$ in $3 \mathrm{~h}$ & 6 \\
\hline black $\mathrm{BiOCl}$ & - & $20 \mathrm{mg} \mathrm{L}^{-1} \mathrm{RhB}$ & $90 \%$ in $50 \mathrm{~min}$ & 7 \\
\hline $\mathrm{BiOCl}(010)$ & 4.3 & $5.0 \times 10^{-5} \mathrm{~mol} \mathrm{~L}^{-1} 2-\mathrm{NAP}$ & $99 \%$ in $100 \mathrm{~min}$ & 8 \\
\hline BiOCl-PT & 56 & 10 ppm RhB & $86 \%$ in $3 \mathrm{~h}$ & 9 \\
\hline $\mathrm{BiOCl}$ & 5.6 & $10 \mathrm{mg} \mathrm{L}^{-1} \mathrm{RhB}$ & $98.1 \%$ in $195 \mathrm{~min}$ & 10 \\
\hline I-doped $\mathrm{BiOCl}$ & - & $60 \mathrm{mg} \mathrm{L}^{-1} \mathrm{RhB}$ & $99.5 \%$ in $60 \mathrm{~min}$ & 11 \\
\hline
\end{tabular}




\section{References}

(1) Huang, H.; Ma, C.; Zhu, Z.; Yao, X.; Liu, Y.; Liu, Z.; Li, C.; Yan, Y. Insights into enhanced visible light photocatalytic activity of t-Se nanorods/BiOCl ultrathin nanosheets 1D/2D heterojunctions. Chem. Eng. J. 2018, 338, 218-229.

(2) Xu, Y.; Lin, D.; Liu, X.; Luo, Y.; Xue, H.; Huang, B.; Chen, Q.; Qian, Q. Electrospun $\mathrm{BiOCl} / \mathrm{Bi}_{2} \mathrm{Ti}_{2} \mathrm{O}_{7}$ nanorod heterostructures with enhanced solar light efficiency in the photocatalytic degradation of tetracycline hydrochloride. Chem CatChem 2018, 10, 2496-2504.

(3) Wang, H.; Yuan, X.; Wu, Y.; Zeng, G.; Dong, H.; Chen, X.; Leng, L.; Wu, Z.; Peng, L. In situ synthesis of $\mathrm{In}_{2} \mathrm{~S}_{3} @ \mathrm{MIL}-125(\mathrm{Ti})$ core-shell microparticle for the Removal of tetracycline from wastewater by integrated adsorption and visible-light-driven photocatalysis. Appl. Catal. B: Environ. 2016, 186, 19-29.

(4) Jiang, L.; Yuan, X.; Zeng, G.; Liang, J.; Wu, Z.; Wang, H.; Zhang, J.; Xiong, T.; Li, H. A facile band alignment of polymeric carbon nitride isotype heterojunctions for enhanced photocatalytic tetracycline degradation. Environ. Sci.: Nano 2018, 5, 2604-2617.

(5) Wang, Q.; Wang, W.; Zhong, L.; Liu, D.; Cao, X.; Cui, F. Oxygen vacancy-rich 2D/2D BiOCl-g- $\mathrm{C}_{3} \mathrm{~N}_{4}$ ultrathin heterostructure nanosheets for enhanced visible-light-driven photocatalytic activity in environmental remediation. Appl. Catal. B: Environ. 2018, 220, 290-302.

(6) Sun, L.; Xiang, L.; Zhao, X.; Jia, C. J.; Yang, J.; Jin, Z.; Cheng, X.; Fan, W. Enhanced Visible-Light Photocatalytic Activity of BiOI/BiOCl Heterojunctions: Key Role of Crystal Facet Combination. ACS Catal. 2015, 5, 3540-3551.

(7) Wu, S.; Sun, W.; Sun, J.; Hood, Z. D.; Yang, S.; Sun, L.; Kent, P. R. C.; Chisholm, M. F. Surface Reorganization Leads to Enhanced Photocatalytic Activity in Defective BiOCl. Chem. Mater. 2018, 30, 5128-5136.

(8) Weng, S.; Pei, Z.; Zheng, Z.; Hu, J.; Liu, P. Exciton-Free, Nonsensitized Degradation of 2-Naphthol by Facet-Dependent BiOCl under Visible Light: Novel Evidence of Surface-State Photocatalysis. ACS Appl. Mater. Interfaces 2013, 5, 12380-12386.

(9) Guerrero, M.; Altube, A.; García-Lecina, E.; Rossinyol, E.; Baró, M. D.; Pellicer, E.; Sort, J. Facile in Situ Synthesis of $\mathrm{BiOCl}$ Nanoplates Stacked to Highly Porous $\mathrm{TiO}_{2}$ : A Synergistic Combination for Environmental Remediation. ACS Appl. Mater. Interfaces 2014, 6, 13994-14000. 
(10) Zhao, S.; Zhang, Y.; Zhou, Y.; Zhang, C.; Sheng, X.; Fang, J.; Zhang, M. Reactable Polyelectrolyte-Assisted Synthesis of $\mathrm{BiOCl}$ with Enhanced Photocatalytic Activity. ACS Sustainable Chem. Eng. 2017, 5, 1416-1424.

(11) Liu, W.; Shang, Y.; Zhu, A.; Tan, P.; Liu, Y.; Qiao, L.; Chu, D.; Xiong, X.; Pan, J. Enhanced performance of doped $\mathrm{BiOCl}$ nanoplates for photocatalysis: understanding from doping insight into improved spatial carrier separation. J. Mater. Chem. A 2017, 5, 12542-12549. 